\section{Population-based screening for ACPAs: a step in the pathway to the prevention of rheumatoid arthritis?}

Anticitrullinated protein/peptide antibodies (ACPAs), in addition to rheumatoid factor (RF), represent a serological hallmark in the diagnosis of rheumatoid arthritis (RA). In this context and with interest I read the recent article by van Zanten et al ${ }^{1}$ summarising the results from the Dutch 'Lifelines Study', a large population-based study of 40132 individuals. In this setting and using an adjusted cut-off for the ACPA assay, the prevalence of ACPAs was $1.0 \%$ and associated older age, female gender, smoking, joint complaints, RA and first-degree relatives with rheumatism.

The early identification of patients in the preclinical phase of RA is of high importance as it became evident during the last decade that early intervention can prevent joint damage in patients with RA. Consequently, several ongoing studies are focused on the prevention of RA based on the treatment of individuals at high risk to develop RA. ${ }^{2}$ All these prevention trials leverage the concept of the 'window of opportunity' to prevent or delay the clinical ravages and attending healthcare expenditures associated with RA. Although a recent trial was unable to prevent the onset of RA (PRAIRI, unpublished data), the study clearly demonstrated that the treatment in the preclinical phase of RA postponed the development of the disease. Most studies aim to treat patients as early as possible, an approach which seems intuitive. However, it is unclear if treatment too early in the clinical course also leads to failure of response. Most conventional approaches to the prevention of RA are based on the concept of restoring the lost tolerance of the immune system.

\section{PRECLINICAL RA}

The risk for the development of RA depends on many factors, which can be divided into two main categories: the modifiable (eg, smoking, behaviour) and the constant risk factors (eg, genetics). ${ }^{3}$ The preclinical phase of RA may be initiated by modification of the risk profile (eg, smoking) and is characterised by break of tolerance as part of the autoimmune processes, leading to increasing joint inflammation and eventually tissue damage and significant morbidity. The development of RA has primarily been linked to factors that affect the gastrointestinal, the respiratory and reticuloendothelial systems. ${ }^{4}$ An additional challenge to identify the right time for treatment is the patient-specific rate of the evolution from preclinical to clinical RA. In some patients, the preclinical phase can take several years, whereas in other patients, this conversion happens in a shorter period of time. Factors contributing, to defining or accelerating this transition are not fully understood.

\section{BIOMARKERS}

Based on all the findings about the possible treatment in the preclinical phase of RA, reliable biomarkers are needed to identify patients who are on the trajectory to develop RA. Once a panel of biomarkers has been found and carefully validated, population screening for evidence-based, effective treatment is a conceivable approach in the efforts to prevent RA. In this context, recently, the combination of ACPAs, RF and anticarbamylated peptide (CarP) autoantibodies ${ }^{5}$ has been shown to provide a very high odds ratio for RA. ${ }^{6}$ Unfortunately, RF and anti-CarP autoantibody data were not available for the population described by van Zanten et al. ${ }^{1}$ As the Lifelines Study is a prospective longitudinal cohort study with a targeted 30-year follow-up, it might be possible to gain follow-up information on this study population. It will be interesting to see which participants will eventually develop RA. In addition, it would be valuable to test the stored serum samples for as many biomarkers as possible in order to identify patients who develop RA in a certain period of time. Lastly, combining biomarker data with clinical parameters might result in sufficient power to precisely predict the development of RA. ${ }^{7}$

Several studies have now repeatedly shown that ACPAs and other biomarkers (eg, autoantibodies, inflammatory proteins, cytokines and microRNA) can antedate the development of RA by many years. ${ }^{78}$ Although these data are intriguing, it would be more valuable to have biomarkers that are able to provide insights into the evolution of RA within the next 6-12 months, corresponding to the so-called window of opportunity. ${ }^{9} 10$ When it comes to population screening, health economics becomes an important factor in the equation. Since the global healthcare expenditures are constantly increasing and reaching non-sustainable thresholds in many jurisdictions, health economic studies of direct and indirect costs will be needed to demonstrate that investments in screening for pre-RA and early interventions or therapies are not outweighed by poor clinical outcomes. Based on remarkable healthcare expenditures on the management of RA, there is a significant opportunity to achieve meaningful savings. ${ }^{2} 1112$

\section{CLINICAL PREVENTION TRIALS}

The design of the different RA prevention trials is rather diverse in terms of the treatment used and the inclusion criteria for the individuals at risk to be treated. ${ }^{2}$ Therapeutic agents range from hydroxychloroquine to biologics (eg, tumour necrosis factoralpha inhibitors). Different treatments surely require also different timings in the preclinical phase of the disease. Lastly, information as to who will develop RA in the distant future might be more useful in case preventive drugs (such as vaccines) become available.

\section{Michael Mahler}

Correspondence to Dr Michael Mahler, Research, Inova Diagnostics, 9900 Old Grove Road, San Diego, CA 92131, USA; mmahler@inovadx.com

Competing interests Employee of Inova Diagnostics selling autoantibody assays. Provenance and peer review Not commissioned; internally peer reviewed.

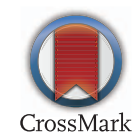

To cite Mahler M. Ann Rheum Dis 2017;76:e42.

Accepted 2 February 2017

Published Online First 8 March 2017

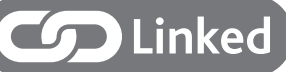

http://dx.doi. org/10.1136/annrheumdis-2017-211256

Ann Rheum Dis 2017;76:e42. doi:10.1136/annrheumdis-2017-211249

REFERENCES

1 van Zanten A, Arends S, Roozendaal C, et al. Presence of anticitrullinated protein antibodies in a large population-based cohort from the Netherlands. Ann Rheum Dis 2017; 0: 1-7.

2 Finckh $\mathrm{A}$, Escher $\mathrm{M}$, Liang $\mathrm{MH}$, et al. Preventive treatments for rheumatoid arthritis: issues regarding patient preferences. Curr Rheumatol Rep 2016;18:51. 
3 Deane KD. Preclinical rheumatoid arthritis (autoantibodies): an updated review. Curr Rheumatol Rep 2014;16:419.

4 Deane KD, El-Gabalawy H. Pathogenesis and prevention of rheumatic disease: focus on preclinical RA and SLE. Nat Rev Rheumato/ 2014;10:212-28.

5 Trouw LA, Mahler M. Closing the serological gap: promising novel biomarkers for the early diagnosis of rheumatoid arthritis. Autoimmun Rev 2012:12:318-22.

6 Shi J, van Steenbergen HW, van Nies JA, et al. The specificity of anti-carbamylated protein antibodies for rheumatoid arthritis in a setting of early arthritis. Arthritis Res Ther 2015;17:339.

7 Rakieh C, Nam JL, Hunt L, et al. Predicting the development of clinical arthritis in anti-CCP positive individuals with non-specific musculoskeletal symptoms: a prospective observational cohort study. Ann Rheum Dis 2015;74:1659-66.
8 Kokkonen $\mathrm{H}$, Mullazehi M, Berglin $\mathrm{E}$, et al. Antibodies of $\lg \mathrm{G}$, IgA and IgM isotypes against cyclic citrullinated peptide precede the development of rheumatoid arthritis. Arthritis Res Ther 2011;13:R13.

9 van Nies JA, Krabben A, Schoones JW, et al. What is the evidence for the presence of a therapeutic window of opportunity in rheumatoid arthritis? A systematic literature review. Ann Rheum Dis 2014;73:861-70.

10 Mankia K, Emery P. A new window of opportunity in rheumatoid arthritis: targeting at-risk individuals. Curr Opin Rheumatol 2016;28:260-6.

11 Anaya JM, Duarte-Rey C, Sarmiento-Monroy JC, et al. Personalized medicine. Closing the gap between knowledge and clinical practice. Autoimmun Rev 2016; 15:833-42.

12 Finckh A, Deane KD. Prevention of rheumatic diseases: strategies, caveats, and future directions. Rheum Dis Clin North Am 2014;40:771-85. 